\title{
Medicina y otras Carreras de la Salud en Chile. Un análisis preliminar
}

\author{
Ernesto Medina L, Ana María Kaempffer R. \\ The Health Careers teaching system \\ in Chile
}

Background: During 2006, Chilean universities had 586,000 students, corresponding to $41 \%$ of the population aged 18 to 23 years. Aim: To evaluate the situation of health care teaching in Chile. Material and Methods: Data from 6,212 careers were elaborated, considering the area of knowledge taught, level of teaching, geography, number and type of students, admission and tuition fees. Results: There are 537 programs for health care teaching in Chile. Of these, 231 are for nursing or dentistry aide personnel. The system has 60,648 matriculated students and it collected a total of US\$250 millions in tuition fees. Only 9,8\% of programs are accreditated. The students who chose health careers had the highest scores in the National University Selection Examination or PSU. Between 2000 and 2006, there was a significant increase in the number of medical students and every year, 1,000 new physicians will receive their title. During 2007, 1,634 new posts were offered in medical schools, 3,873 in nursing and 5,671 for kinesiology. Conclusions: There is a disharmonic growth of University teaching in Chile, that will solve historical deficits of professionals such as nurses but will introduce important changes in Chilean medicine (Rev Méd Chile 2007; 135: 1346-54).

(Key words: Education, medical, undergraduate; Health care; Students, health occupations; Students, medical)

Recibido el 11 de abril de 2007. Aceptado el 21 de septiembre de 2007.

Escuela de Salud Pública, Facultad de Medicina, Universidad de Chile. Santiago de Chile.

$\mathrm{E}^{\mathrm{n}}$ n las últimas décadas, Chile ha experimentado una llamativa mejoría del nivel educacional de su población. Los alumnos matriculados en la enseñanza básica y media constituyen $93 \%$ de los niños de 6 a 18 años. El ingreso a la universidad se percibe como ventajoso por razones económicas, laborales y sociales y ello ha generado una importante presión para expandir la educación superior. En el año 2006 las universidades chilenas

Correspondencia a: Dr. Ernesto Medina L. Medinacelli 1327, Las Condes, Santiago de Chile. Teléfonos: Casa 2121613; Oficina 6782138. E mail: emedina@med.uchile.cl tuvieron 586.000 alumnos, que corresponden a $41 \%$ de la población de 18 a 23 años.

El país se ha dado un sistema de educación superior caracterizado por la facilidad de creación de universidades privadas autónomas, lo que ha llevado a la existencia de unas 50 universidades ${ }^{1}$, en un proceso no planificado, dentro de un sistema competitivo que en el año 2006 recaudó el equivalente a 1.500 millones de dólares. Esta recaudación provino del pago de matrículas y aranceles por los alumnos, sumándosele otras fuentes de recursos de diferente tipo, incluyendo ayudas y aportes fiscales.

El conjunto de universidades ofreció en el año 2007 un total de 189.319 vacantes para el primer 
año de diversas carreras profesionales y técnicas. Dada la importancia que tiene la educación universitaria y el volumen que ha adquirido, parece necesario conocer algunas características actuales de la educación superior en nuestro país. Esta comunicación revisa y analiza preliminarmente el sistema, con énfasis en lo relativo a las Carreras del área de la Salud.

\section{MATERIAL Y MÉTODO}

Se ha contado con datos del Instituto Nacional de Estadística (INE) en cuanto a población, distribución geográfica y por edades ${ }^{2}$, datos técnicos del Consejo Superior de Educación ${ }^{2,3}$ y otras publicaciones sobre el tema ${ }^{4-7}$. Se consideraron los datos de 6.212 Carreras de Universidades, Institutos Profesionales y Centros de Formación Técnica. Se identificó en cada Carrera el área del conocimiento, ubicación geográfica, alumnos matriculados, vacantes de primer año, número de semestres de la Carrera, horario, puntaje de la Prueba de Selección Universitaria (PSU) de los alumnos, valores de matrícula y arancel anual, año de inicio del programa y nivel de enseñanza. Los datos se organizaron por áreas del conocimiento, nivel de enseñanza, ubicación geográfica (regiones y ciu- dades), matrícula total y vacantes ofrecidas en el primer año para la Carrera, antigüedad del programa, existencia o no de acreditación, valores de la matrícula y arancel anual. La información se presenta organizada como listados simples, construyendo sistemas ordenados que permiten comparar algunas características de la educación superior en Chile, particularmente en las Carreras del área de la Salud.

\section{RESULTADOS}

Número y nivel de las carreras. De acuerdo a la información disponible, el número de Carreras ofrecidas en 2007 para todas las áreas fue de 6.212 de las cuales $53 \%$ son Carreras universitarias clásicas, 6\% para ingenieros de ejecución y 41\% para técnicos del nivel superior. En el área de la salud se incluyen auxiliares de enfermería y técnicos en odontología. La mitad de las Carreras ofrecidas corresponden a tecnologías de la ingeniería y administración, y un número importante para formar profesores (Tabla 1).

Matrícula y aranceles. En el momento actual, dos elementos centrales para el cálculo financiero son el número de alumnos matriculados y el valor de

Tabla 1. El sistema de educación superior en Chile. C arreras ofrecidas según áreas y niveles de enseñanza. 2007

\begin{tabular}{|lcr|}
\hline & No de carreras & $\%$ \\
\hline Total & 6.212 & 100,0 \\
Tecnologías & 1.559 & 25,1 \\
Administración & 1.319 & 21,2 \\
Educación & 907 & 14,6 \\
Ciencias Sociales & 549 & 8,8 \\
Salud & 538 & 8,6 \\
Arte y Arquitectura & 421 & 6,8 \\
Agropecuaria & 280 & 4,5 \\
Humanidades & 117 & 1,9 \\
Ciencias & 116 & 1,6 \\
Tipos de carreras & & \\
Profesionales & & 53,2 \\
Ingeniería de Ejecución & 3.307 & 6,0 \\
Técnicos de nivel superior & 372 & 40,8 \\
\hline
\end{tabular}


matrícula y aranceles que pagan cada alumno. De acuerdo a nuestros cálculos, los valores promedio de aranceles del total de Carreras del país es de $\$ 1.497 .000$, oscilando desde $\$ 1.360 .000$ en Historia hasta $\$ 2.840 .000$ para Medicina que, junto a Odontología, tienen las cifras más altas.

Los recursos generados son elevados, como se aprecia en la Tabla 2 que muestra los valores de las Carreras de la Salud. Las cifras de ingresos de las instituciones resultan del producto de los valores medios de matrículas y aranceles de cada Carrera en el país y el número de alumnos matriculados en la Carrera. De acuerdo a este cálculo, las instituciones de enseñanza superior registraron en el año 2006, por concepto de matrícula y aranceles, una cantidad cercana a US\$1.500 millones. El área de la salud, con 10 Carreras, totaliza $\$ 123.838 .000 .000$, cifra que constituye $15,7 \%$ del total. En este cálculo no hemos incluido $\$ 18.946$ millones pagados por alumnos auxiliares de enfermería y técnicos en odontología. En áreas afines a Salud destacan los valores encontrados para las Carreras de Psicología, con \$38.015 millones y Medicina Veterinaria, con \$22.676 millones. Algunas Carreras generan gran cantidad de recursos por el gran número de alumnos matriculados, que superan 20.000 alumnos en Carreras como Derecho, Ingeniería Comercial, Agronomía, Psicología y Pedagogía en Educación Media.

Acreditación de las Carreras. En la educación superior chilena una baja proporción de los programas se encuentra acreditada institucionalmente, lo que se ha cumplido sólo en 219 programas $(3,5 \%)$. Las proporciones más altas de acreditación se observan en las áreas Agropecuaria $(20,5 \%)$ y Salud $(9,8 \%)$ y las más bajas en Humanidades (2,1\%) y Administración (3,9\%). En la Carrera de Medicina, hasta 2006 estaban acreditadas sólo 5 de 23 Escuelas: Universidad de Chile, Pontificia Universidad Católica de Chile, Universidad de Concepción, Universidad de La Frontera y Universidad Austral de Chile. En el año 2007 obtuvieron acreditación las Escuelas de Medicina de la Universidad Mayor y la Universidad de Santiago de Chile, dando a la fecha un total de 7 Escuelas acreditadas entre 23 existentes. En el área de la Salud las mayores tasas de acreditación aparecen en las Carreras de Medicina, Obstetricia, Enfermería y Odontología (Tabla 3).

Tabla 2. Ingresos económicos provenientes del pago de matrícula y arancel anual por los alumnos de las C arreras de la Salud. C hile, 2006

\begin{tabular}{|lcc|}
\hline Carreras & Alumnos matriculados & Ingresos (millones \$) \\
\hline Medicina & 9.503 & 31.265 \\
Odontología & 6.941 & 22.764 \\
Kinesiología & 12.503 & 22.375 \\
Enfermería & 12.407 & 17.515 \\
Tecnología Médica & 3.996 & 7.927 \\
Química y Farmacia & 3.664 & 7.445 \\
Nutrición & 456 & 7.396 \\
Fonoaudiología & 3.008 & 4.475 \\
Obstetricia & 2.676 & 2.654 \\
Terapia Ocupacional & 1.435 & 1.546 \\
& & \\
Auxiliares de enfermería & 4.843 & 4.202 \\
Técnicos en odontología & 2.989 & 2.252 \\
& & \\
Psicología & 22.323 & 38.015 \\
Derecho & 29.202 & 59.203 \\
Ingeniería Comercial & 21.633 & 57.419 \\
Agronomía & 25.712 & 50.909 \\
\hline
\end{tabular}


Tabla 3. Acreditación de las C arreras de la Salud. Chile, 2006

\begin{tabular}{|lrc|}
\hline Carreras & Número & \% acreditado \\
\hline Química y Farmacia & 9 & 44,4 \\
Medicina & 23 & 30,4 \\
Obstetricia & 11 & 18,1 \\
Enfermería & 65 & 13,6 \\
Odontología & 23 & 13,0 \\
Tecnología & 21 & 9,5 \\
Nutrición & 41 & 7,3 \\
Terapia Ocupacional & 14 & 7,1 \\
Kinesiología & 71 & 5,6 \\
Fonoaudiología & 22 & 1,5 \\
& & \\
Carreras de la Salud & 300 & 11,3 \\
Otras carreras & 5.912 & 3,1 \\
Total & 6.212 & 3,5 \\
\hline
\end{tabular}

Vacantes de primer año en 2007 y puntajes de ingreso. Para el año 2007, las universidades y centros de enseñanza superior ofrecieron 120.956 vacantes (Tabla 4) siendo las Carreras más ofrecidas las de Derecho (7.733 plazas en la Carrera de Derecho más 1.475 para otras Carreras del área del Derecho, particularmente Criminología), Pedagogía Básica (6.543), Ingeniería en Computación (6.103), Kinesiología (5.671) y Diseño (5.635). Las probabilidades de ingreso están vinculadas al puntaje en la Prueba de Selección Universitaria (PSU). En la Tabla 5 aparecen los puntajes más bajos con los cuales entraron alumnos en la Carrera correspondiente, en el año 2006, en la Universidad de Chile. Cuatro Carreras del área de la Salud se ubican dentro de las cinco que recibieron alumnos con puntaje inferior más alto (Medicina, Odontología, Kinesiología, Fonoaudiología), contrastando con Enfermería cuyo puntaje la ubica en el lugar $21^{\circ}$.

Tabla 4. Vacantes de primer año en las C arreras de la Salud y otras C arreras profesionales. Chile, 2007 (no incluye $C$ arreras para técnicos de nivel superior)

\begin{tabular}{|lrr|}
\hline Tipo de carreras & Número & $\%$ del total \\
\hline Carreras de la Salud & 19.060 & 15,2 \\
Kinesiología & 5.189 & 4,2 \\
Enfermería & 3.823 & 3,2 \\
Odontología & 1.914 & 1,7 \\
Nutrición & 1.960 & 1,6 \\
Medicina & 1.634 & 1,4 \\
Tecnología Médica & 1.227 & 1,7 \\
Química y Farmacia & 625 & 0,6 \\
Obstetricia & 596 & 0,6 \\
Fonoaudiología & 897 & 0,7 \\
Terapia Ocupacional & 595 & 0,6 \\
Otras Carreras & & \\
Educación y Pedagogías & 27.114 & 22,2 \\
Tecnologías & 16.561 & 13,7 \\
Administración & 12.697 & 10,5 \\
Ciencias Sociales & 12.456 & 10,3 \\
Arquitectura y Artes & 11.197 & 9,3 \\
Derecho & 9.208 & 7,6 \\
Ciencias & 5.173 & 4,4 \\
Humanidades & 3.464 & 3,0 \\
Total de Carreras & 116.930 & 100,0 \\
\hline
\end{tabular}


Tabla 5. Puntajes más bajos en la Prueba de Selección U niversitaria (PSU) que fueron aceptados para ingresar en las C arreras de la Salud. C hile, 2007

\begin{tabular}{|lll|}
\hline Puntaje PSU & Carreras de la Salud & Otras Carreras \\
\hline 727 & Medicina & \\
690 & Odontología & \\
680 & Kinesiología & Antropología \\
646 & Fonoaudiología & Licenciatura Biología \\
$630-639$ & Terapia Ocupacional & Ingeniería Comercial \\
& Nutrición & Sociología \\
$620-629$ & Tecnología Médica & Derecho \\
& Química y Farmacia & \\
$610-619$ & Medicina Veterinaria & \\
$543-605$ & Obstetricia & \\
\hline
\end{tabular}

Crecimiento de los programas en las Carreras de la Salud. Sólo 12 de ellos se crearon antes de 1935. Hubo un número importante de nuevos programas en Medicina a partir del año 1998. En el período 2000-2007 se iniciaron entre $81 \%$ y $89 \%$ de las nuevas Carreras de Fonoaudiología, Terapia Ocupacional y Odontología, 50\% de Medicina y 58\% de Enfermería (Tabla 6).

Tamaño probable de las promociones médicas. En la Tabla 7 aparece el tamaño probable de las promociones anuales, considerando el número de

Tabla 6. Antigüedad de las C arreras de la Salud. Porcentaje de programas iniciados en el período 2000-2007

\begin{tabular}{|lc|}
\hline Carreras & Porcentajes \\
\hline Fonoaudiología & 91 \\
Nutrición & 90 \\
Terapia Ocupacional & 88 \\
Kinesiología & 87 \\
Enfermería & 75 \\
Odontología & 70 \\
Tecnología Médica & 62 \\
Química y Farmacia & 44 \\
Medicina & 34 \\
Obstetricia & 30 \\
\hline
\end{tabular}

alumnos que ya están dentro de la Carrera (matrícula total) y el número de años de estudio. A las cifras resultantes hay que restarles el porcentaje de abandono de los estudios, fenómeno que ocurre preferentemente en los primeros dos años de estudios y que ha sido estimado entre $5 \%$ y $8 \%$ para Medicina y Odontología y entre $20 \%$ y $25 \%$ para otras Carreras de la Salud.

Otras características. El 93\% de los programas se hace en jornada diurna, siendo de $80 \%$ para las auxiliares de enfermería. El promedio nacional de duración de las Carreras es de 7,52 semestres.

Carreras de la Salud en las regiones geográficas chilenas. Existe enseñanza superior desde Arica hasta Magallanes (Tabla 8), con algunas zonas de alta densidad, particularmente Concepción con 54 alumnos universitarios por 100.000 habitantes (Tabla 8).

Universidades y Centros de Formación Técnica. La Tabla 9 muestra que 35 instituciones participaron como formadoras de profesionales de la Salud en el año 2006. Diecisiete de ellas corresponden a universidades y 18 a institutos profesionales y centros de formación técnica. El 59\% de los alumnos matriculados pertenecen a universidades del Consejo de Rectores y $41 \%$ restante corresponde a universidades privadas. La proporción de 
Tabla 7. Tamaño probable de las promociones anuales de las Carreras de la Salud en el próximo quinquenio

\begin{tabular}{|lrrrr|}
\hline Carreras & (a) & (b) & (c) & (d) \\
\hline Kinesiologia & 12.583 & 5 & 2.566 & 2.013 \\
Enfermería & 12.407 & 5 & 2.481 & 1.985 \\
Medicina & 9.502 & 7 & 1.357 & 1.290 \\
Odontología & 5.941 & 6 & 1.068 & 966 \\
Nutrición & 4.456 & 5 & 891 & 713 \\
Tecnología Médica & 3.996 & 5 & 799 & 639 \\
Química y Farmacia & 3.664 & 5 & 732 & 585 \\
Fonoaudiología & 3.008 & 5 & 601 & 481 \\
Obstetricia & 2.654 & 5 & 530 & 424 \\
Terapia Ocupacional & 1.435 & 5 & 287 & 230 \\
\hline
\end{tabular}

(a) Total de matriculados. (b) Duración de la carrera (años). (c) Promoción anual. (d) Ajustada por deserción

\section{Tabla 8. Alumnos de las Carreras de la Salud según ciudad en que cursan sus estudios. Tasas por 1.000 habitantes. Año 2006}

\begin{tabular}{|lrc|}
\hline Ciudades & Número & Tasa x 1.000 hab \\
\hline Santiago & 36.442 & 8 \\
Concepción & 11.269 & 54 \\
Valparaíso & 4.900 & 17 \\
Viña del Mar & 4.230 & 12 \\
Temuco & 4.141 & 11 \\
Talca & 3.315 & 16 \\
Antofagasta & 3.217 & 12 \\
Arica & 2.003 & 10 \\
Valdivia & 1.887 & 8 \\
Puerto Montt & 1.465 & 6 \\
Iquique & 800 & 8 \\
La Serena & 493 & 5 \\
Osomo & 780 & 5 \\
Rancagua & 723 & 3 \\
\hline
\end{tabular}

estas últimas es alta en Medicina (54\%) y oscila entre $54 \%$ y $67 \%$ en otras Carreras.

La Carrera de Medicina. La Tabla 10 muestra los principales atributos de la Carrera, que se imparte en 23 centros universitarios. La mitad de ellos iniciaron su actividad después de 1998. En el año 2006 las vacantes de primer año alcanzaron a
1.634 y el total de alumnos matriculados fue de 9.503. En catorce Escuelas de Medicina las promociones anuales no superan 60 alumnos. En cuatro Escuelas (Universidad de Chile, Pontificia Universidad Católica de Chile, Universidad de Concepción y Universidad de Los Andes) el último alumno ingresado a primer año tuvo más de 700 puntos en la PSU.

La enseñanza de Enfermería. Esta Carrera constituye una área de gran expansión en años recientes, contabilizándose 60 centros de formación para enfermeras, con 12.407 alumnas y 96 institutos profesionales y centros de formación técnica para auxiliares de enfermería, con 11.193 estudiantes.

Formación de personal auxiliar en Salud. De las 528 Carreras de la Salud del sistema chileno, $59 \%$ corresponden a profesionales y $41 \%$ a técnicos, como auxiliares de enfermería (165 Carreras) y auxiliares dentales (82 Carreras). Algunas instituciones tienen gran responsabilidad en la formación de personal auxiliar, como ocurre con el Instituto Profesional AIEP con 46 Carreras, el Centro de Formación Técnica Santo Tomás (28), el Centro de Formación Técnica INACAP (28) y el Instituto Profesional del Valle Central (18), que simultáneamente son también las mayores instituciones formadoras de auxiliares dentales. 
Tabla 9. Principales instituciones formadoras de profesionales de la salud. N úmero total de alumnos en Carreras de la Salud, en M edicina y Enfermería. 2006

\begin{tabular}{|lcrr|}
\hline Instituciones* & Total & Medicina & Enfermería \\
\hline U. Andrés Bello & 5.794 & 429 & 757 \\
U. de Chile & 5.204 & 1.459 & 731 \\
U. de Concepción & 3.455 & 782 & 501 \\
U. Mayor & 3.089 & 414 & 94 \\
U. de Valparaíso & 2.858 & 418 & 434 \\
U. San Sebastián & 2.132 & 821 & 543 \\
U. Católica de Chile, Santiago & 1.807 & 769 & 549 \\
U. Austral & 1.489 & 359 & 359 \\
U. de Santiago & 1.408 & 592 & - \\
U. Diego Portales & 1.358 & 268 & 348 \\
U. Santo Tomás & 1.298 & & 840 \\
U. de Viña del Mar & 1.124 & 290 & \\
U. de La Frontera & 1.118 & 420 & 292 \\
U. de Los Andes & 1.103 & 481 & 707 \\
U. de Antofagasta & 1.081 & 374 & \\
U. Finis Terrae & 1.057 & 279 & \\
U. Católica del Maule & 1.033 & & \\
\hline
\end{tabular}

(*) Incluye 181 carreras de la salud.

\section{DisCUSIÓN}

En relación a la pregunta básica de este estudio, de cómo es la enseñanza superior en Chile, particularmente en las Carreras de la Salud, los datos recogidos nos sugieren las siguientes respuestas:

1. La enseñanza superior del país se desarrolló en el año 2007 a través de 6.212 Carreras con 586.000 alumnos, lo que constituye un importante esfuerzo. Dos tercios de la tarea universitaria se destina a programas en tecnologías de ingeniería, de administración y a formación de profesores de enseñanza media, lo que revela que estas actividades tienen hoy una clara prioridad. Hay un significativo esfuerzo de formación de técnicos de nivel superior. Las Carreras de la Salud realizan 8,6\% de la tarea global de acuerdo al número de alumnos matriculados.

2. El área de la Salud tiene 60.000 alumnos en formación, con 9.500 estudiantes de Medicina y 6.900 de Odontología. Las Carreras que constituyen novedad por su gran número de alumnos son Kinesiología y Enfermería, con 12.000 alumnos cada una.

3. El incremento del número de alumnos ha sido muy grande en otras áreas. Las Carreras de Derecho, Ingeniería Comercial y Agronomía tienen más de 20.000 alumnos matriculados.

4. Las Carreras de la Salud generaron en el año 2006, por concepto de matrícula y aranceles, la cifra de 123.838 millones de pesos, equivalentes a 250 millones de dólares. El total de valores de las matrículas y aranceles de la educación superior fue en 2006 de 1.500 millones de dólares, que equivalen a 1,1\% del PIB.

5. No hay elementos suficientes para juzgar la calidad de la enseñanza universitaria chilena. Una muy baja proporción de las Carreras $(3,5 \%)$ están acreditadas, lo que se eleva a $11,3 \%$ en las Carreras de la Salud. Las mayores proporciones de acreditación se registran en las Carreras de Química y Farmacia, Medicina, Obstetricia, Enfermería y Odontología. Sólo tres universidades chilenas (Universidad de 
Tabla 10. La C arrera de M edicina en las U niversidades C hilenas. Año 2006

\begin{tabular}{|c|c|c|c|c|c|c|}
\hline Universidades & (a) & (b) & (c) & (d) & (e) & (f) \\
\hline Iquique & 60 & 162 & 600 & 2,9 & 470 & 2004 \\
\hline Antofagasta & 60 & 420 & 642 & 2,8 & 1.176 & 1996 \\
\hline Católica del Norte & 55 & 215 & 659 & 2,8 & 602 & 2003 \\
\hline Valparaíso-S. Felipe & 44 & 74 & 671 & 2,6 & 192 & 2005 \\
\hline Valparaíso & 54 & 428 & 692 & 2,6 & 1.112 & 1961 \\
\hline Viña del Mar & 80 & 290 & & 2,7 & 783 & 2003 \\
\hline \multicolumn{7}{|l|}{ Andrés Bello } \\
\hline (Sede Viña del Mar) & 40 & 72 & & 3,5 & 212 & 2005 \\
\hline (Sede Santiago) & 100 & 429 & & 4,2 & 1.844 & 2001 \\
\hline Santiago & 61 & 592 & 686 & 2,4 & 1.425 & 1993 \\
\hline Desarrollo & 85 & 405 & 686 & 4,1 & 1.666 & 2002 \\
\hline Católica de Chile & 90 & 769 & 770 & 3,7 & 2.865 & 1930 \\
\hline Finis Terrae & 45 & 259 & 604 & 4,0 & 1.036 & 2002 \\
\hline Diego Portales & 68 & 266 & 650 & 4,0 & 1.072 & 2002 \\
\hline Mayor & 60 & 414 & 680 & 4,1 & 759 & 1998 \\
\hline U. de Chile & 160 & 1.459 & 727 & 2,8 & 4.085 & 1842 \\
\hline Los Andes & 80 & 414 & 680 & 3,9 & 1.887 & 1991 \\
\hline Católica del Maule & 61 & 171 & 662 & 2,7 & 461 & 2004 \\
\hline Concepción & 198 & 782 & 710 & 3,4 & 2.659 & 1924 \\
\hline San Sebastián & 125 & 543 & 593 & 3,9 & 2.117 & 1994 \\
\hline Sma. Concepción & 48 & 342 & & 3,0 & 1.026 & 2004 \\
\hline La Frontera & 56 & 420 & 686 & 2,5 & 1.050 & 1970 \\
\hline Austral & 45 & 659 & 662 & 2,8 & 1.005 & 1967 \\
\hline S. Sebastián-P. Montt & 50 & 146 & 574 & 3,5 & 511 & 2002 \\
\hline Total Chile & 1.685 & 9.503 & 524 & & 35.214 & \\
\hline
\end{tabular}

Vacantes de primer año. (b) Matrícula total. (c) Puntaje mínimo de ingreso. (d) Arancel (millones de \$). (e) Ingreso anual (millones de \$). (f) Año de inicio de la Carrera de Medicina.

Chile, Pontificia Universidad Católica de Chile y Universidad Adolfo Ibáñez) aparecen en los listados chino y británico clasificadas entre "las 500 mejores universidades del mundo".

6. El área de la Salud ofreció $15,6 \%$ de las vacantes universitarias de primer año en 2007. La mayoría de los programas de enseñanza actualmente vigentes son programas nuevos iniciados entre los años 2000 y 2007.

7. Las Carreras de la Salud atraen a los alumnos con mejores resultados en la PSU: Medicina, Odontología, Kinesiología y Fonoaudiología concentran a 4 de los 5 mejores resultados de esta prueba.

8. El gran número de estudiantes de Kinesiología y Enfermería probablemente resolverá el déficit crónico de estos profesionales. La existencia de 9.500 alumnos de Medicina, que equivalen a la mitad de los médicos activos en Chile, implica que en los próximos 7 años habrá promociones anuales de 1.000 médicos, a los que se sumarán el importante número de médicos extranjeros que están emigrando al país.

9. Al proceso de formación de nuevos profesionales de la Salud están contribuyendo todas las ciudades chilenas de mayor tamaño y unas 35 instituciones públicas y privadas de educación superior.

10. La Carrera de Medicina se dicta en 23 centros universitarios, ubicados en nueve de las trece regiones geográficas del país, revelando un alto grado de descentralización. 
11. La baja tasa de acreditación de las Escuelas de Medicina puede deberse al corto tiempo que llevan desde su creación: muchas todavía no han graduado su primera promoción de profesiona-

\section{REFERENCIAS}

1. CRUZ-Coke R. Las universidades chilenas. 19812004. Rev Méd Chile 2004; 136: 1543-8.

2. InSTItUTO Nacional de Estadística. Compendio estadístico 2005.

3. Consejo Superior de Educación. Resultados de la Prueba de Selección Universitaria (2006).

4. Consejo Superior de Educación. Índices 2007 de Carreras Profesionales y Técnicas. les. Sin embargo, una nueva Ley de la República obliga a someterse a acreditación institucional a todas las Carreras de Educación y Salud, proceso que debe estar iniciado en el año 2008.

5. Goic A. Seminario sobre formación de médicos en la actualidad en Chile. Rev Méd Chile 1999; 131: 2001-10.

6. Rosselot E. La globalización de la educación superior europea. Rev Méd Chile 2001; 133: 833-40.

7. GonZÁLEZ LE. La repitencia y duración de la educación superior chilena. Publicación UNESCO 2005.

8. Medina E, KaEMPFFer AM. Necesidad de médicos en Chile. Rev Méd Chile 1998; 116: 389-94. 